Internat. J. Math. \& Math. Sci.

Vol. 24, No. 4 (2000) 277-281

S0161171200003926

(c) Hindawi Publishing Corp.

\title{
FANTASTIC FILTERS OF LATTICE IMPLICATION ALGEBRAS
}

\author{
YOUNG BAE JUN
}

(Received 22 September 1999)

\begin{abstract}
The notion of a fantastic filter in a lattice implication algebra is introduced, and the relations among filter, positive implicative filter, and fantastic filter are given. We investigate an equivalent condition for a filter to be fantastic, and state an extension property for fantastic filter.
\end{abstract}

Keywords and phrases. Lattice implication algebra, filter, implicative filter, positive implicative filter, fantastic filter.

2000 Mathematics Subject Classification. Primary 03G10, 06B10, 54E15.

1. Introduction. In order to research the logical system whose propositional value is given in a lattice, $\mathrm{Xu}$ [5] proposed the concept of lattice implication algebras, and discussed their some properties. Also, in [6], Xu and Qin discussed the properties of lattice $H$ implication algebras, and gave some equivalent conditions about lattice $H$ implication algebras. For the general development of lattice implication algebras, the filter theory plays an important role as well as ideal theory. Xu and Qin [7] introduced the notion of filters in a lattice implication algebra, and investigated their properties. In [2], we gave an equivalent condition of a filter, and provided some equivalent conditions that a filter is an implicative filter, and using this result an extension property for implicative filter is constructed. Jun et al. [4] introduced the concepts of a positive implicative filter and an associative filter in a lattice $H$ implication algebra. They proved that (i) every positive implicative filter is an implicative filter, and (ii) every associative filter is a filter. They also provided equivalent conditions for both a positive implicative filter and an associative filter. In [3], Jun et al. defined an $L I$-ideal of a lattice implication algebra and showed that every $L I$-ideal is a lattice ideal. They gave an example that a lattice ideal may not be an $L I$-ideal, and showed that every lattice ideal is an $L I$-ideal in a lattice implication algebra. They discussed the relationship between filters and $L I$-ideals, and studied how to generate an $L I$-ideal by a set. Moreover they constructed the quotient structure by using an $L I$-ideal, and studied the properties of $L I$-ideals related to implication homomorphisms. In this paper, the notion of a fantastic filter in a lattice implication algebra is introduced, and then we give the relations among filter, positive implicative filter and fantastic filter. We investigate an equivalent condition for a filter to be fantastic, and state an extension property for fantastic filter.

2. Preliminaries. By a lattice implication algebra we mean a bounded lattice $(L, \vee$, $\wedge, 0,1)$ with order-reversing involution ", " and a binary operation " $\rightarrow$ " satisfying the 
following axioms:

(I1) $x \rightarrow(y \rightarrow z)=y \rightarrow(x \rightarrow z)$,

(I2) $x \rightarrow x=1$,

(I3) $x \rightarrow y=y^{\prime} \rightarrow x^{\prime}$,

(I4) $x \rightarrow y=y \rightarrow x=1 \Rightarrow x=y$,

(I5) $(x \rightarrow y) \rightarrow y=(y \rightarrow x) \rightarrow x$,

(L1) $(x \vee y) \rightarrow z=(x \rightarrow z) \wedge(y \rightarrow z)$,

(L2) $(x \wedge y) \rightarrow z=(x \rightarrow z) \vee(y \rightarrow z)$,

for all $x, y, z \in L$.

Note that the conditions (L1) and (L2) are equivalent to the conditions

(L3) $x \rightarrow(y \wedge z)=(x \rightarrow y) \wedge(x \rightarrow z)$, and

(L4) $x \rightarrow(y \vee z)=(x \rightarrow y) \vee(x \rightarrow z)$, respectively.

EXAMPLE 2.1. Let $L:=\{0, a, b, c, 1\}$. Define the partially ordered relation on $L$ as $0<a<b<c<1$, and define $x \wedge y:=\min \{x, y\}, x \vee y:=\max \{x, y\}$ for all $x, y \in L$ and ", "and " $\rightarrow$ " as follows:

TABLE 2.1.

\begin{tabular}{|c||c|}
\hline$x$ & $x^{\prime}$ \\
\hline \hline 0 & 1 \\
\hline$a$ & $c$ \\
\hline$b$ & $b$ \\
\hline$c$ & $a$ \\
\hline 1 & 0 \\
\hline
\end{tabular}

\begin{tabular}{|c||c|c|c|c|c|}
\hline$\rightarrow$ & 0 & $a$ & $b$ & $c$ & 1 \\
\hline \hline 0 & 1 & 1 & 1 & 1 & 1 \\
\hline$a$ & $c$ & 1 & 1 & 1 & 1 \\
\hline$b$ & $b$ & $c$ & 1 & 1 & 1 \\
\hline$c$ & $a$ & $b$ & $c$ & 1 & 1 \\
\hline 1 & 0 & $a$ & $b$ & $c$ & 1 \\
\hline
\end{tabular}

Then $\left(L, \vee, \wedge,,^{\prime} \rightarrow\right)$ is a lattice implication algebra.

In the sequel the binary operation " $\rightarrow$ " will be denoted by juxtaposition. We can define a partial ordering " $\leq$ " on a lattice implication algebra $L$ by $x \leq y$ if and only if $x y=1$.

In a lattice implication algebra $L$, the following hold (see [5]):

(1) $0 x=1,1 x=x$, and $x 1=1$.

(2) $x^{\prime}=x 0$.

(3) $x y \leq(y z)(x z)$.

(4) $x \vee y=(x y) y$.

(5) $\left((y x) y^{\prime}\right)^{\prime}=x \wedge y=\left((x y) x^{\prime}\right)^{\prime}$.

(6) $x \leq y$ implies $y z \leq x z$ and $z x \leq z y$.

(7) $x \leq(x y) y$.

In what follows, $L$ denotes a lattice implication algebra unless otherwise specified.

Definition 2.2 (Xu et al. [7]). A subset $F$ of $L$ is called a filter of $L$ if it satisfies:

(F1) $1 \in F$,

(F2) $x \in F$ and $x y \in F$ imply $y \in F$ for all $x, y \in L$.

A subset $F$ of $L$ is called an implicative filter of $L$ if it satisfies (F1) and 
(F3) $x(y z) \in F$ and $x y \in F$ imply $x z \in F$ for all $x, y, z \in L$.

Proposition 2.3 (Jun [2, Proposition 3.2]). Every filter $F$ of L has the following property:

$$
x \leq y \text { and } x \in F \text { imply } y \in F .
$$

DeFinITION 2.4 (Jun et al. [4]). A subset $F$ of $L$ is called a positive implicative filter of $L$ if it satisfies (F1) and

(F4) $x((y z) y) \in F$ and $x \in F$ imply $y \in F$ for all $x, y, z \in L$.

Proposition 2.5 (Jun [4, Theorem 3.1]). Every positive implicative filter $F$ of $L$ is a filter.

Proposition 2.6 (Jun [4, Theorem 3.3]). Let $F$ be a filter of L. Then $F$ is a positive implicative filter of $L$ if and only if

(F5) $(x y) x \in F$ implies $x \in F$ for all $x, y \in L$.

Proposition 2.7 (Jun [2, Theorem 3.4]). Let $F$ be a non-empty subset of L. Then $F$ is a filter of $L$ if and only if it satisfies: for all $x, y \in F$ and $z \in L$,

(F6) $x \leq y z$ implies $z \in F$.

\section{Fantastic filters}

DEFinITION 3.1. A non-empty subset $F$ of $L$ is called a fantastic filter of $L$ if it satisfies (F1) and

(F7) $z(y x) \in F$ and $z \in F$ imply $((x y) y) x \in F$ for all $x, y, z \in L$.

EXAMPLE 3.2. Let $L:=\{0, a, b, c, d, 1\}$ be a set with Figure 3.1 as a partial ordering. Define a unary operation ", " and a binary operation denoted by juxtaposition on $L$ as follows (Tables 3.2 and 3.3, respectively):

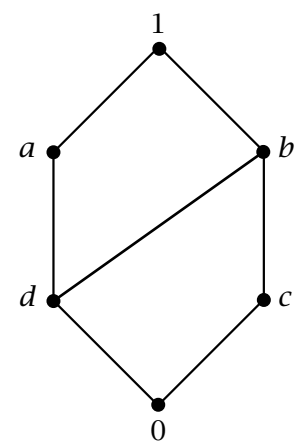

FIGURE 3.1.

Define $\vee$ - and $\wedge$-operations on $L$ as follows:

$$
x \vee y:=(x y) y, \quad x \wedge y:=\left(\left(x^{\prime} y^{\prime}\right) y^{\prime}\right)^{\prime},
$$


TABLE 3.2.

\begin{tabular}{|c||c|}
\hline$x$ & $x^{\prime}$ \\
\hline \hline 0 & 1 \\
\hline$a$ & $c$ \\
\hline$b$ & $d$ \\
\hline$c$ & $a$ \\
\hline$d$ & $b$ \\
\hline 1 & 0 \\
\hline
\end{tabular}

TABLE 3.3.

\begin{tabular}{|c||c|c|c|c|c|c|}
\hline & 0 & $a$ & $b$ & $c$ & $d$ & 1 \\
\hline \hline 0 & 1 & 1 & 1 & 1 & 1 & 1 \\
\hline$a$ & $c$ & 1 & $b$ & $c$ & $b$ & 1 \\
\hline$b$ & $d$ & $a$ & 1 & $b$ & $a$ & 1 \\
\hline$c$ & $a$ & $a$ & 1 & 1 & $a$ & 1 \\
\hline$d$ & $b$ & 1 & 1 & $b$ & 1 & 1 \\
\hline 1 & 0 & $a$ & $b$ & $c$ & $d$ & 1 \\
\hline
\end{tabular}

for all $x, y \in L$. Then $L$ is a lattice implication algebra. One can see that $F:=\{b, c, 1\}$ is a fantastic filter of $L$.

THEOREM 3.3. Every fantastic filter of $L$ is a filter.

Proof. Let $F$ be a fantastic filter of $L$ and let $z x \in F$ and $z \in F$. Then $z(1 x) \in F$ and $z \in F$. It follows from (F7) that $x=((x 1) 1) x \in F$ so that $F$ is a filter.

We now give an equivalent condition for a filter to be a fantastic filter.

THEOREM 3.4. A filter $F$ of $L$ is fantastic if and only if it satisfies:

(F8) $y x \in F$ implies $((x y) y) x \in F$ for all $x, y \in L$.

Proof. Assume that $F$ is a fantastic filter of $L$ and let $x, y \in L$ be such that $y x \in F$. Then $1(y x)=y x \in F$ and $1 \in F$. It follows from (F7) that $((x y) y) x \in F$. Conversely let $F$ be a filter of $L$ satisfying (F8) and let $x, y, z \in L$ be such that $z(y x) \in F$ and $z \in F$. Then $y x \in F$ by (F2) and hence $((x y) y) x \in F$ by (F8). Therefore $F$ is a fantastic filter of $L$.

THEOREM 3.5. Every positive implicative filter of $L$ is fantastic.

Proof. Let $F$ be a positive implicative filter of $L$. Then $F$ is a filter of $L$ (see Proposition 2.5). Let $x, y \in L$ be such that $y x \in F$. It is sufficient to show that $((x y) y) x \in F$. Since $x \leq((x y) y) x$, we get $(((x y) y) x) y \leq x y$. Putting $a=$ $((x y) y) x$, we obtain

$$
\begin{aligned}
(a y) a & =((((x y) y) x) y)(((x y) y) x) \\
& \geq(x y)(((x y) y) x)=((x y) y)((x y) x) \geq y x .
\end{aligned}
$$


It follows from Proposition 2.3 that ( $a y) a \in F$ so, from Proposition 2.6, that $a \in F$, i.e., $((x y) y) x \in F$. Hence $F$ is a fantastic filter of $L$.

OPEN PROBLEM. Does the converse of Theorem 3.5 hold?

THEOREM 3.6 (extension property for fantastic filter). Let $F$ and $G$ be filters of $L$ such that $F \subseteq G$. If $F$ is fantastic, then so is $G$.

Proof. Let $x, y \in L$ be such that $y x \in G$. Then $y((y x) x)=(y x)(y x)=1 \in F$. Since $F$ is fantastic, it follows from Theorem 3.4 that

$$
((((y x) x) y) y)((y x) x) \in F
$$

so that $(y x)(((((y x) x) y) y) x) \in F \subseteq G$. Since $y x \in G$, therefore $((((y x) x) y) y) x \in$ G. But

$$
\begin{aligned}
(((((y x) x) y) y) x)(((x y) y) x) & \geq((x y) y)((((y x) x) y) y) \\
& \geq(((y x) x) y)(x y) \geq x((y x) x) \\
& =(y x)(x x)=(y x) 1=1 .
\end{aligned}
$$

Using Proposition 2.7, we get ( $(x y) y) x \in G$. Hence, by Theorem 3.4, $G$ is a fantastic filter of $L$.

\section{REFERENCES}

[1] Y. B. Jun, The prime filter theorem of lattice implication algebras, Int. J. Math. Math. Sci., to appear.

[2] __ Implicative filters of lattice implication algebras, Bull. Korean Math. Soc. 34 (1997), no. 2, 193-198. MR 98g:03142. Zbl 876.03035.

[3] Y. B. Jun, E. H. Roh, and Y. Xu, LI-ideals in lattice implication algebras, Bull. Korean Math. Soc. 35 (1998), no. 1, 13-24. MR 98j:06002. Zbl 903.03037.

[4] Y. B. Jun, Y. Xu, and K. Qin, Positive implicative and associative filters of lattice implication algebras, Bull. Korean Math. Soc. 35 (1998), no. 1, 53-61. MR 98j:06003. Zbl 903.03038.

[5] Y. Xu, Lattice implication algebras, J. Southwest Jiaotong Univ. (1993), no. 1, 20-27. Zbl 784.03035.

[6] Y. Xu and K. Y. Qin, Lattice H implication algebras and lattice implication algebra classes, J. Hebei Mining and Civil Engineering Institute (1992), no. 3, 139-143.

[7] _ _ On filters of lattice implication algebras, J. Fuzzy Math. 1 (1993), no. 2, 251-260. MR 94b:06016. Zbl 787.06009.

Young Bae Jun: Department of MATHematics Education, GyeONGSANG NATional UniVERSITY, CHINJU 660-701, KOREA

E-mail address: ybjun@nongae.gsnu.ac.kr 


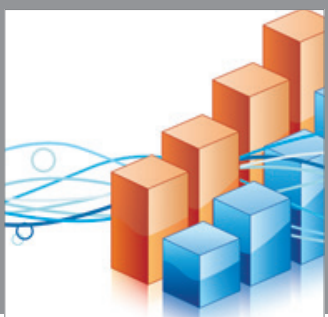

Advances in

Operations Research

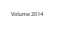

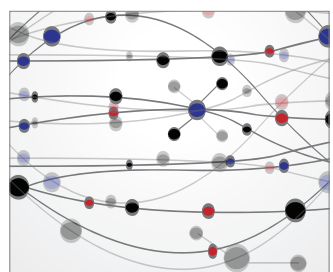

\section{The Scientific} World Journal
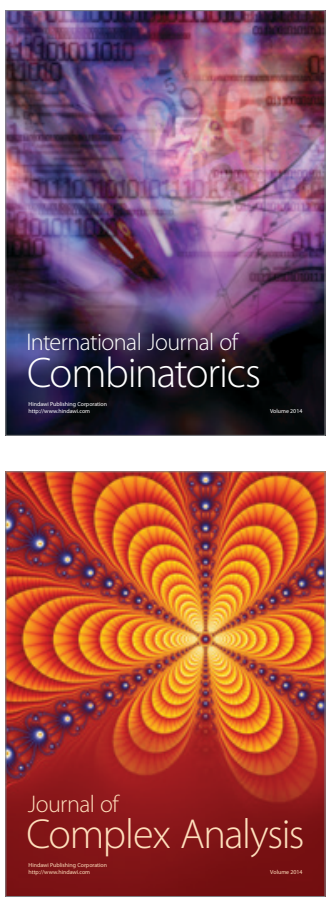

International Journal of

Mathematics and

Mathematical

Sciences
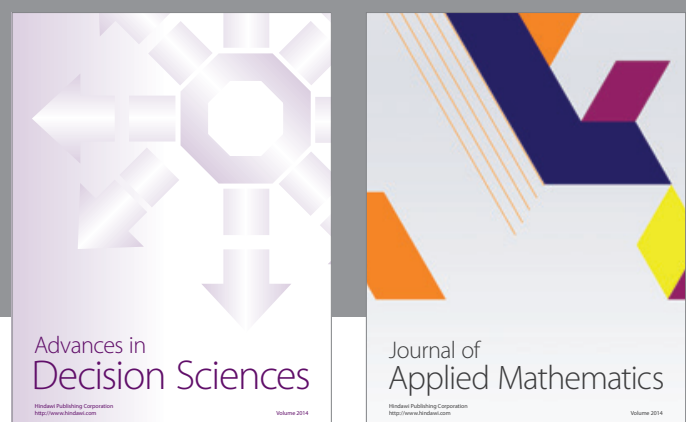

Journal of

Applied Mathematics
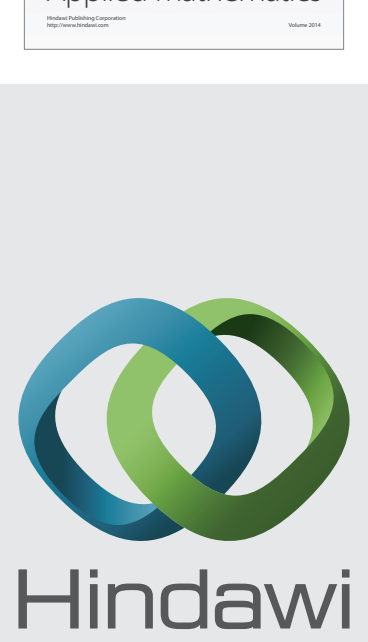

Submit your manuscripts at http://www.hindawi.com
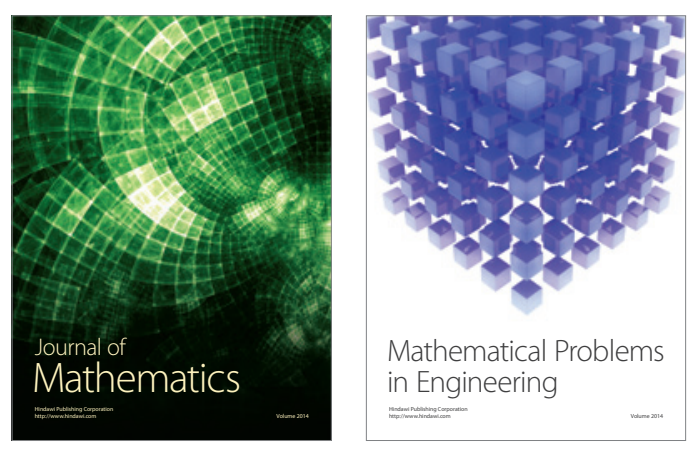

Mathematical Problems in Engineering
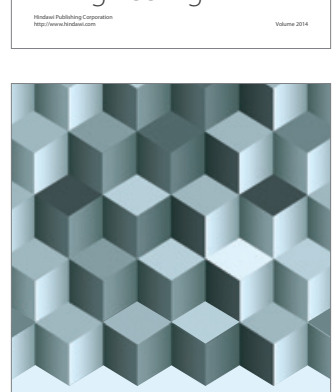

Journal of

Function Spaces
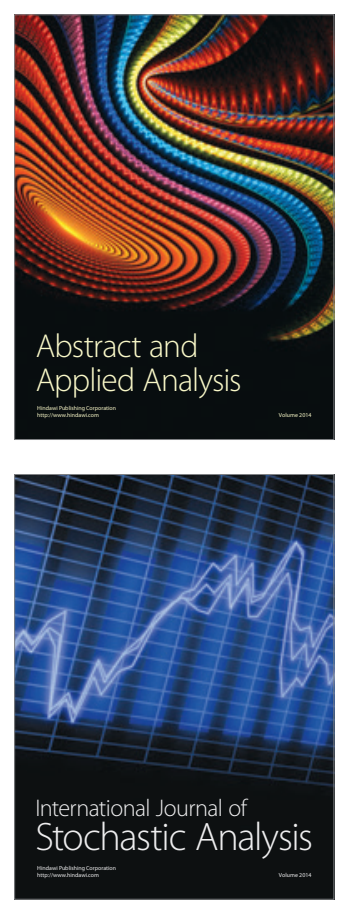

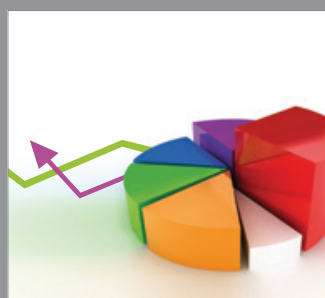

ournal of

Probability and Statistics

Promensencen
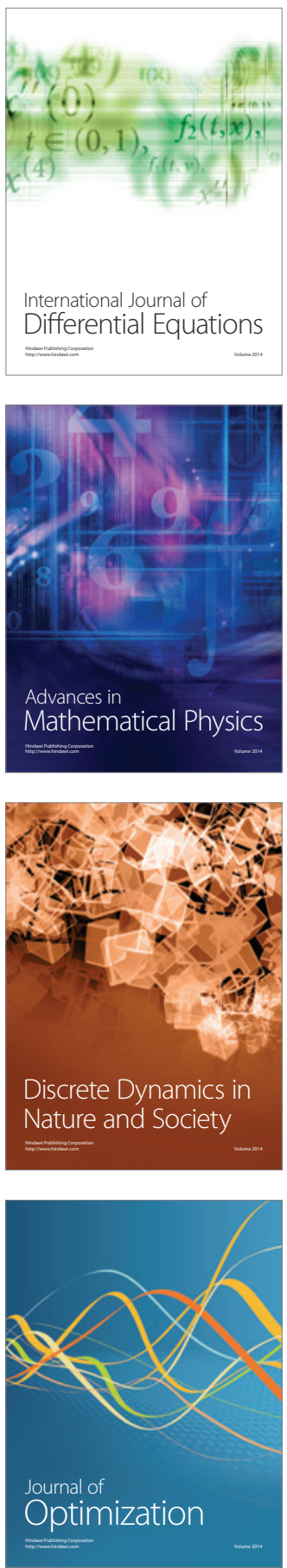